\title{
Does prolonged infusion time really improve the efficacy of meropenem therapy? A prospective study in critically ill patients
}

\section{Yi Chang Zhao}

Second Xiangya Hospital https://orcid.org/0000-0002-9651-4457

\section{Yang Zou}

Xiangtan Central Hospital

\section{Yi Wen Xiao}

Second Xiangya Hospital

\section{Feng Wang}

Second Xiangya Hospital

\section{Bi Kui Zhang}

Second Xiangya Hospital

\section{Da Xiong Xiang}

Second Xiangya Hospital

\section{Feng Yu}

China Pharmaceutical University

Hong Luo

Second Xiangya Hospital

Miao Yan ( $\square$ yanmiao@csu.edu.cn )

\section{Research Article}

Keywords: Meropenem, Population pharmacokinetics, Pharmacodynamics, Critically ill patients, Pneumonia

Posted Date: July 13th, 2021

DOI: https://doi.org/10.21203/rs.3.rs-471732/v3

License: (c) (i) This work is licensed under a Creative Commons Attribution 4.0 International License. Read Full License

Version of Record: A version of this preprint was published at Infectious Diseases and Therapy on November 6th, 2021. See the published version at https://doi.org/10.1007/s40121-021-00551-2. 


\section{Abstract}

Background: Meropenem is a carbapenem antibiotic that has demonstrated excellent in vitro activity against gram-negative clinical isolates and is commonly used in critically ill patients. This study aimed to find the pharmacokinetic/ pharmacodynamic of meropenem in critically ill patients and whether prolonged injection duration is really beneficial to meropenem therapy.

Method: We included 209 samples in 64 patients in this prospective study. PPK analysis and Monte Carlo dosing simulations were developed using Phoenix.

Results: A two-compartment model described the data adequately. Clearance (CL), volume (V), clearance of peripheral compartment $\left(\mathrm{CL}_{2}\right)$, volume of peripheral compartment $\left(\mathrm{V}_{2}\right)$ were $6.15 \mathrm{~L} / \mathrm{h}, 2.83 \mathrm{~L} / \mathrm{h}, 17.40 \mathrm{~L}$, and $17.48 \mathrm{~L}$, respectively. Creatinine clearance and uric acid were significant covariates. Patients with creatinine clearance of $60 \mathrm{ml} / \mathrm{min}$ or less and uric acid greater than $400 \mu \mathrm{mol} / \mathrm{l}$ could achieve the target > $90 \%$ under the minimum inhibitory concentration (MIC) of $8 \mathrm{mg} / \mathrm{L}$, even with the administration dose of $500 \mathrm{mg} / 8 \mathrm{~h}$ with a 2-h infusion. Prolonging the infusion time significantly improved the therapeutic effect when MIC $\varangle$. However, for the pharmacodynamic (PD) effects of $100 \% \mathrm{fT}>\mathrm{MIC}$ and $100 \% \mathrm{fT}>4 \mathrm{MIC}$, no significant statistical difference was observed in critically ill patients.

Conclusions: Critically ill patients with lower creatinine clearance and higher uric acid levels were likely to need a lower dosage of meropenem. Prolonged infusion time were not always beneficial for those who need a higher therapeutic target ( $100 \% \mathrm{fT}>\mathrm{MIC}, 100 \% \mathrm{fT}>4 \mathrm{MIC})$ or with MIC $4 \mathrm{mg} / \mathrm{L}$. Increasing dose or alternative therapeutic strategies may be required for critically ill patients with drug-resistant or severe infections. The study is of great significance to guide the rational use of meropenem in critically ill patients.

Trial registration: The trial was registered in the China Clinical Trial (ChiCTR1900020672). Registered on 12 January 2019.

\section{Key Points}

This study provides reliable evidence of the efficacy and safety of meropenem in critically ill patients. It is the first time to find that uric acid level significantly impacts meropenem use and to assess the achievement of various PK/PD targets (40\% time free concentration above MIC (fT>MIC), $100 \% \mathrm{fT}>\mathrm{MIC}$ and $100 \% \mathrm{fT}>4 \mathrm{MIC}$ ) with MIC values ranging from $1-8 \mathrm{mg} / \mathrm{L}$ in critically ill patients with severe pneumonia. During clinical empirical therapy, dose adjustment based on creatinine clearance and uric acid appears to be reasonable. Patients with a lower level of creatinine clearance and a high uric acid level tend to require lower dosages. There was significant clinical benefit from prolonged infusion time when $\mathrm{MIC} \geq 4 \mathrm{mg} / \mathrm{L}$. Increasing dose or alternative therapeutic strategies may be required for critically ill patients with drug-resistant or severe infections who require higher therapeutic targets.

\section{Introduction}


Severe pneumonia is a risk factor for in-hospital mortality ${ }^{1-4}$. In recent years, carbapenems have been widely used in patients and are considered the last line of defense in treating gram-negative bacterial infections ${ }^{5-7}$. Meropenem is a second-generation carbapenem antibiotic. Unlike the first generation, the 1$\beta$ methyl modification of the chemical structure enhances the stability of the drug to renal dehydropeptidase $\mathrm{I}^{8,9}$. It is also a broad-spectrum antimicrobial used as empirical or directed therapy in critically ill patients. Meropenem shows time-dependent antibacterial activity and is characterized by linear pharmacokinetics in vivo; higher doses correspond to higher peak and trough concentrations ${ }^{10,11}$. In healthy volunteers, the elimination half-life of meropenem in plasma was about 1 hour ${ }^{12,13}$.

Severe pathophysiological changes in critical illness can lead to dramatically altered antimicrobial pharmacokinetics (PK). In populations such as children, elderly, and obese, those with severe burns, those treated with continuous renal replacement therapy, and patients on extracorporeal membrane oxygenation, meropenem shows significant individual differences in plasma concentrations and pharmacokinetic parameters ${ }^{14-18}$. These effects are related to the time that the free concentration is maintained above the minimum inhibitory concentration (MIC) (fT > MIC), at least $40 \%{ }^{19}$. Several clinical studies suggest that $100 \% \mathrm{fT}>$ MIC in plasma is associated with better therapeutic effects ${ }^{20-24}$. Additionally, it has been widely reported that prolonged injection duration can improve the therapeutic effect of meropenem ${ }^{25}, 26$. Nevertheless, it remains unclear whether standard meropenem dosing regimens achieve this target in critically ill patients. Therefore, this study aimed to measure the meropenem pharmacokinetic in critically ill patients and improve pharmacokinetic/pharmacodynamic and patient outcomes.

\section{Materials And Methods}

\section{Study design and patients}

This prospective study was conducted at the Department of Respiratory and Intensive Care Unit, the Second Xiang-ya Hospital of Central South University, between January and December 2019. The Ethics Committee of our hospital approved the study (number 2019-005) that was registered as a China Clinical Trial (ChiCTR1900020672).

Patients treated with meropenem and admitted to the Department of Respiratory and Critical Care Medicine were eligible. Written informed consent was obtained from all participants. Inclusion criteria were as follows: (i) severe lung infection; (ii) clear indications for the use of meropenem; (iii) the time of continuous medication exceeded two days; (iv) at least one steady-state plasma concentration could be obtained; (v) age > 18 years, and (vi) gram-negative bacilli were isolated from specimen culture. Exclusion criteria were as follows: (i) pregnancy and lactation; (ii) allergy to carbapenems; (iii) concomitant uses of sodium valproate; (iv) isolation of gram-positive cocci, viruses, or fungi before enrollment; and (v) incomplete dosing information or clinical data. 
Based on the standard recommendations for meropenem use, the conventional dosage regimen was 500 $\mathrm{mg} / 8 \mathrm{~h}$ or $1000 \mathrm{mg} / 8 \mathrm{~h}$, two times/8 h, and continuous infusion for $30 \mathrm{~min}, 1 \mathrm{~h}$ or $2 \mathrm{~h}$. From the electronic medical record information system, we recorded demographic information, clinical data, and laboratory test results using a standardized data collection form on the day of serum sampling. The endogenous creatinine clearance rate was calculated using the Cockcroft-Gault formula27, 28.

According to the MICs of bacteria to meropenem in our hospital, various meropenem MICs $(1,2,4$, and 8 $\mathrm{mg} / \mathrm{L}$ ) were evaluated using the following PK/PD targets: $40 \% \mathrm{fT}$ MIC, $100 \% \mathrm{fT} \mathrm{MIC}$, and $100 \% \mathrm{fT} 4 \mathrm{MIC}$ 23,24 .

\section{Sampling and assays}

Meropenem serum concentrations were measured using automatic two-dimensional high-performance liquid chromatography (Demeter Instrument Co., Ltd., Hunan, China). The first-dimensional chromatographic column was an Aston SNCB $(4.6 \times 50 \mathrm{~mm}, 5 \mu \mathrm{m})$, and the second-dimensional chromatographic column was an Aston SBN $(4.6 \times 200 \mathrm{~mm}, 5 \mu \mathrm{m}){ }^{29,30}$. There was an excellent linear relationship between peak area and the concentration range of 0.78 to $58.52 \mu \mathrm{g} / \mathrm{mL}$. The lower limit of detection and the lower limit of quantification were $0.04 \mu \mathrm{g} / \mathrm{mL}$ and $0.1 \mu \mathrm{g} / \mathrm{mL}$, respectively. The intraday precision, inter-day precision, and accuracy were $1.21 \%-2.58 \%, 0.83 \%-1.80 \%$, and $100.51 \%-$ $101.69 \%$, respectively. The extraction recovery of the high, medium, and low concentrations were $99.47 \%$, $97.77 \%$, and $97.23 \%$, respectively.

\section{Pharmacokinetic study}

The PK model of meropenem in critically ill patients was developed using Phoenix NLME software (Version 8.1, Pharsight, A Certara Company, USA). Serum meropenem concentrations were fitted to a twocompartment model using the logarithmic additive residual. The first-order conditional estimationextended least-squares method was used to estimate model parameters. The goodness of fit and visual predictive check (VPC) were used to evaluate the model. Objective function values (OFV) were used to compare the model fit. Covariates were retained in the model if the additional covariates were significant at a P-value of $0.01(\triangle \mathrm{OFV}>6.635)$. VPC was used to evaluate the goodness of fit ${ }^{12,31-34}$.

\section{Probability of target attainment.}

We use Monte Carlo simulations $(n=3,000)$ to determine the probability of target attainment (PTA) with different significant covariates. Meropenem doses of $500 \mathrm{mg}, 1,000 \mathrm{mg}$, and 2,000 mg given intravenously every $8 \mathrm{~h}$ (q8h) with a duration of $0.5 \mathrm{~h}, 2 \mathrm{~h}$, and $4 \mathrm{~h}$ were simulated at different levels of selected covariates. The PTA was calculated after three days of therapy. The MIC at which PTA was equal to $90 \%$ was derived to enable a numeric comparison among the regimens ${ }^{16,18,35}$. MIC values were selected for the most common value of pathogenic bacterias such as Enterobacter cloacae, Pseudomonas aeruginosa, Klebsiella pneumoniae, and Acinetobacter baumannii obtained from our 
hospital. PTA was calculated for single doses of $500 \mathrm{mg}, 1000 \mathrm{mg}$, and $2000 \mathrm{mg}$. The therapeutic target adopted the effect of $40 \% \mathrm{fT}>\mathrm{MIC}, 100 \% \mathrm{fT}>\mathrm{MIC}$, and $100 \% \mathrm{fT}>4 \mathrm{MIC}^{19,23,24}$.

\section{Statistical analysis.}

Continuous variables are expressed as means (standard deviations [SD]) or medians (interquartile ranges) depending on the normality of distribution. Enumeration data were expressed as absolute numbers and relative frequencies. The Kolmogorov-Smirnov, and Shapiro-Wilk tests were used to test for normality. A two-sided P-value of $<0.05$ was considered statistically significant. One-way analysis of variance was used to test the differences in selected significant covariate groups. All analyses were performed using IBM SPSS Statistics version 25 (IBM, New York, NY). Figures were generated using Phoenix NLME and Graphpad Prism version 8 (San Diego, CA, USA).

\section{Results}

\section{Demographic and clinical data of study patients}

Sixty-four patients were enrolled in this prospective study. A total of 210 meropenem plasma samples were obtained; $73.43 \%$ of the patients were male, with an average weight of $62.5 \mathrm{~kg}$. The average age was 63.5 years, and the mean Acute Physiology and Chronic Health Evaluation (APACHE) score was 17.2. More details about the demographic and clinical characteristics are shown in Table 1.

Table 1 Demographic and laboratory data 


\begin{tabular}{|c|c|}
\hline \multicolumn{2}{|l|}{ Demographic and clinical data ${ }^{a}$} \\
\hline Gender(male), N(\%) & $47(73.43 \%)$ \\
\hline Age (year), mean $\pm S D$ & $63.5 \pm 14.5$ \\
\hline Weight $(\mathrm{kg})$, mean \pm SD & $62.5 \pm 13.3$ \\
\hline $\begin{array}{l}\text { Score of APACHE } \square^{\mathrm{a}}, \text { mean } \pm \text { SD } \\
\mathrm{PaO} 2 / \mathrm{FiO} 2(\%), \text { mean } \pm \text { SD }\end{array}$ & $\begin{array}{r}17.2 \pm 7.8 \\
162.6 \pm 79.3\end{array}$ \\
\hline \multicolumn{2}{|l|}{ Type of pneumonia, N (\%) } \\
\hline Community Acquired Pneumonia, N(\%) & $20 \square 31.25 \% \square$ \\
\hline Nosocomial pneumonia, N(\%) & $42 \square 65.63 \% \square$ \\
\hline Others, $\mathrm{N}(\%)$ & $2 \square 3.1 \% \square$ \\
\hline \multicolumn{2}{|l|}{ Other clinical data } \\
\hline White blood cell $\left(10^{\wedge} 9 / \mathrm{L}\right),(\mathrm{IQR})$ & $10.3[6.3-15.5]$ \\
\hline Red blood cell $\left(10^{\wedge 12} / \mathrm{L}\right)$, mean \pm SD & $3.4 \pm 0.8$ \\
\hline Platelets $\left(10^{\wedge} 9 / \mathrm{L}\right),(\mathrm{IQR})$ & $162.0[99.8-314.3]$ \\
\hline Hemoglobin $(\mathrm{g} / \mathrm{L})$, mean \pm SD & $102.8 \pm 25.6$ \\
\hline Alanine aminotransferase $(\mathrm{U} / \mathrm{L})$ & $32.6[17.1-53.3]$ \\
\hline Aminotransferase (U/L), (IQR) & $43.4[30.6-72.3]$ \\
\hline Albumin $(\mathrm{g} / \mathrm{L})$, mean $\pm \mathrm{SD}$ & $27.1 \pm 4.4$ \\
\hline Urea nitrogen (mmol/L), (IQR) & $8.3[5.7-16.2]$ \\
\hline Creatinine $(\mu \mathrm{mol} / \mathrm{L}),(\mathrm{IQR})$ & 69.2[53.4-154.0] \\
\hline Uric acid $(\mu \mathrm{mol} / \mathrm{L}),(\mathrm{IQR})$ & $187.0[133.0-345.7]$ \\
\hline $\begin{array}{l}\text { Cockcroft-Gault Creatinine clearances }(\mathrm{mL} / \mathrm{min}) \text {, (IQR) } \\
\text { Inflammatory indicators }\end{array}$ & $75.9[40.3-100.1]$ \\
\hline Procalcitonin ( $\mu \mathrm{g} / \mathrm{L})$, (IQR) & $0.6[0.1-3.5]$ \\
\hline C-reactive protein $(\mathrm{mg} / \mathrm{L}),(\mathrm{IQR})$ & $124.0[78.4-214.8]$ \\
\hline Erythrocyte sedimentation rate $(\mathrm{mm} / \mathrm{h}),(\mathrm{IQR})$ & $74.5[40.5-101.0]$ \\
\hline Temperature $\left({ }^{\circ} \mathrm{C}\right)$, (IQR) & $38.1[37.2-38.7]$ \\
\hline
\end{tabular}

$\mathrm{SD}$, standard deviation; IQR, interquartile range

${ }^{\mathrm{a}}$ Calculated at the beginning of ICU admission

Meropenem sensitivity test was performed in $71.86 \%$ of the patients, most of whom had more than one kind of pathogen. A total of 80 meropenem MIC values were collected, $53.75 \%$ of which were above 8 $\mathrm{mg} / \mathrm{L}$. The detection rate of $A$. baumannii was the highest (21 [26.3\%]). Notably, the MIC values for this pathogen were above $8 \mathrm{mg} / \mathrm{L}$, suggesting resistance to meropenem. Notably, 45 (56.3\%) of the gramnegative bacteria were multi-drug-resistant. Microbiological data and distributions of MICs are displayed in Table 2.

Table 2 Microbiological data 


\begin{tabular}{lr}
\hline Microbiological data a & \\
\hline No. of patients collected MIC (mg/L), N(\%) & $46(71.86 \%)$ \\
& $24(30 \%)$ \\
& $10(12.5 \%)$ \\
& $2(2.5 \%)$ \\
& $1(1.3 \%)$ \\
Microbiological specie, N(\%) & $43(53.75 \%)$ \\
Baumanii & $46(71.86 \%)$ \\
Klebsiella pneumoniae & $21(26.3 \%)$ \\
Pseudomonas aeruginosa & $19(23.8 \%)$ \\
Stenotrophomonas maltophilia & $11(13.8 \%)$ \\
Enterobacter cloacae & $7(8.8 \%)$ \\
Alcaligenes & $4(5 \%)$ \\
Klebsiella oxytoca & $3(3.8 \%)$ \\
Ralstonia pickettii & $2(2.5 \%)$ \\
Burkholderia onion & $2(2.5 \%)$ \\
Other Gram-negative bacteria & $2(2.5 \%)$ \\
MDR Gram-negative bacteria & $4(5 \%)$ \\
Sensitive Gram-negative bacteria & $45(56.3 \%)$ \\
\hline
\end{tabular}

MDR, multidrug-resistant.

\section{Pharmacokinetic model}

A total of 210 meropenem plasma concentrations were included in the population analysis. The meropenem PPK was best described by a two-compartment linear model with first-order elimination. A stepwise method was used to determine all the covariates that may affect the pharmacokinetic parameters. For covariates, we selected gender, age, body weight, APACHE score, Cockcroft-Gault CLCR (CG-CLCR), white blood cell, red blood cell, platelets, hemoglobin, alanine aminotransferase, aminotransferase, albumin, urea nitrogen, and uric acid. Inflammatory indicators were also included in the covariate selection. Despite various covariates having relationships with the estimated clearance, they were not included in the final model. Uric acid was finally found to be closely connected to meropenem V2 and CL. CG-CLCR was closely connected to CL. These two factors for meropenem V2 and CL improved the model fit best. When they were added to the model, the log-likelihood value from the previous model was significantly improved $(P<0.01)$. The covariate model was as follows: 


$$
\begin{aligned}
& \mathrm{CL}=\operatorname{tvCL} \times(\text { Uric Acid } / 245.24)^{-0.537} \times(C G-C L C R / 80.67)^{0.636} \times \exp (\eta \mathrm{CL}) \\
& \mathrm{V}=\mathrm{tvV} \times \exp (\eta \mathrm{V}) \\
& \mathrm{CL}=\mathrm{tv} C L_{2} \exp \left(\eta C L_{2}\right) \\
& V_{2}=\mathrm{tv} V_{2}(\text { Uric Acid } / 245.24)^{1.799} \times \exp \left(\eta V_{2}\right)
\end{aligned}
$$

In these equations, CG-CLCR was calculated using the Cockcroft-Gault equation; tvCL is the typical value of meropenem clearance, $\mathrm{CL}$ is the population parameter of meropenem clearance, $\mathrm{tvV}$ is the typical value of volume in the central compartment, $V_{2}$ is the population parameter estimate for the volume of the peripheral compartment, and $\eta \mathrm{CL}, \eta \mathrm{V}, \eta C L_{2}$, and $\eta V_{2}$ are the inter-individual random effects of the parameter.

The parameters for the basic and final covariate model are shown in Table 3. Individual and population predicted serum meropenem concentrations vs. observed concentrations are shown in Fig. 1. The distribution of conditional weighted residuals is presented in Fig. 2. The values of conditional weighted residuals were between -2 to 2 . Both plots indicated the fitting advantages of the final model. The final covariate model was used for Monte Carlo dosing simulations.

Table 3 Final parameter estimates of final and basic meropenem model

\begin{tabular}{lll}
\hline Parameter & Final model & Basic model \\
\hline -2LL & 274.55 & 350.42 \\
Parameter value & & \\
V (liter) & 17.40 & 11.83 \\
V $_{2}$ (liter) & 17.48 & 10.41 \\
CL(liter/h) & 6.15 & 6.35 \\
CL (liter/h) & 2.83 & 11.25 \\
CV c of parameter value(\%) & & \\
V (liter) & 7.25 & 23.59 \\
V (liter) & 23.19 & 23.17 \\
CL (liter/h) & 10.38 & 10.34 \\
CL (liter/h) & 26.73 & 39.41 \\
Shrinkage (\%) & 13.93 & 19.07 \\
\hline
\end{tabular}

-2LL, -2 log likelihood; CL, population central clearance of meropenem; V, population volume of distribution in the central compartment; CL2, clearance of meropenem from the central to the peripheral compartment; CV, coefficient of variation.

\section{Simulations}


Monte Carlo simulations and meropenem probabilities of target attainment for various CG-CLCR values, uric acid values, dosage regimens, and MICs with a duration of $0.5 \mathrm{~h}, 2 \mathrm{~h}$, and $4 \mathrm{~h}$ are presented in an additional file. Details are shown in Additional file 1-3. MIC values listed in the tables were chosen according to the sensitivity of pathogenic bacteria to meropenem at our hospital. We found that at the lowest dosage ( $500 \mathrm{mg} / \mathrm{q} 8 \mathrm{~h}$ ), patients with uric acid levels of $>400 \mathrm{~mol} / \mathrm{L}$ can achieve an optimal PTA ( of $40 \% \mathrm{fT}>$ MIC for isolates with MICs of $8 \mathrm{mg} / \mathrm{L}$ with a duration of $4 \mathrm{~h}$. However, those with uric acid levels $>40 \mathrm{~mol} / \mathrm{L}$ and CG-CLCR of $120 \mathrm{ml} / \mathrm{min}$ could not achieve optimal PTA ( of $40 \% \mathrm{fT}>\mathrm{MIC}$ for isolates with MICs of $1 \mathrm{mg} / \mathrm{L}$, even with the highest dosage of $2000 \mathrm{mg} / 8 \mathrm{~h}$ with a duration of $0.5 \mathrm{~h}$. Moreover, the numbers of targeted PTA for the three infusion time groups were $55.56 \%, 62.96 \%$, and $77.78 \%$, respectively. These findings suggest that to achieve an optimal PTA, a prolonged infusion time, higher dose, or alternative administration protocol is needed for this cohort. Notably, patients with uric acid levels of $800 \mu \mathrm{mol} / \mathrm{L}$ and CG-CLCR of $30 \mathrm{ml} / \mathrm{min}$ can achieve optimal PTA ( for all targeted therapeutic effects, including 100\% fT > 4MIC for isolates with MICs of $8 \mathrm{mg} / \mathrm{L}$ using a dosage of 2000 $\mathrm{mg} / 8 \mathrm{~h}$ with infusion durations of $2 \mathrm{~h}$ and $4 \mathrm{~h}$. The low uric acid group of $40 \mathrm{~mol} / \mathrm{L}$ failed to achieve the PK/PD target of $100 \% \mathrm{fT}>\mathrm{MIC}$ and $100 \% \mathrm{fT}>4 \mathrm{MIC}$ for all the simulated dosing regimens. In general, high levels of CG-CLCR and low levels of uric acid were associated with lower PTA. Detailed influences of creatinine and uric acid on PTA are shown in Fig. 3. Patients with high creatinine clearance rates were likely to have lower PTA $(P=0.003)$, while those with high levels of uric acid were likely to have higher PTA $($ P $<$ (Fig. 3).

We analyzed the PTA obtained from the simulation result and found that the infusion times of $2 \mathrm{~h}$ and 4 $\mathrm{h}$ appeared to have a higher PTA value than $0.5 \mathrm{~h}$ on average $(P=0.047, \mathrm{Fig} .4)$. However, the number of targeted PTA ( ) showed no significant difference among the three groups $(P=0.6847)$. Given this surprising result, various MICs and therapeutic targets were analyzed. The results are presented in Fig. 5. We found that the duration of infusion affected the improvement of PTA (Fig. 5). For the target of $40 \% \mathrm{fT}$ MIC, PTA was significantly different among the three groups of simulated data when MIC $<\mathrm{mg} / \mathrm{L}(\mathrm{P}<$ $0.05)$. Under these circumstances, PTA could be improved by prolonged infusion time. The difference in PTA was close to significant when MIC was $4 \mathrm{mg} / \mathrm{L}(\mathrm{P}=0.0568)$. Notably, the P-value of the three groups was 0.234 for the target of $40 \% \mathrm{fT}$ MIC when MIC was $8 \mathrm{mg} / \mathrm{L}$ This finding suggests that, for drugresistant bacteria with high MICs, prolonged infusion time does not improve PTA level. There was no significant difference in PTA among the three groups for the targets $100 \%$ fT MIC and $100 \%$ fT $4 \mathrm{MIC}$, even though MIC was $1 \mathrm{mg} / \mathrm{L}$.

\section{Discussion}

I We developed a PPK model of meropenem in patients with severe pulmonary infection. In the previous literature, correlations of antibiotic $\mathrm{CL}$ with creatinine clearance were often reported. To the best of our knowledge, our study is the first to determine that uric acid is a significant covariate describing the pharmacokinetic parameters of meropenem. See Table S1-S3 in the electronic supplementary material for details. These tables display the PTAs for all simulated dosage regimens using $40 \% \mathrm{fT}>\mathrm{MIC}, 100 \% \mathrm{fT}>$ MIC, and 100\% fT > 4MIC pharmacodynamic (PD) thresholds, respectively. According to further PTA 
analysis, we observed that higher levels of CG-CLCR and lower levels of uric acid were associated with the lower achievement of PK/PD targets for critically ill patients. Many studies found that the characteristics of meropenem pharmacokinetics could be described in different populations using a two-compartment model, which is consistent with the results of our study $16,17,36-38$. Adela et al. found that the administration of $2000 \mathrm{mg} / 8 \mathrm{~h}$ of meropenem as a continuous infusion allowed higher serum meropenem concentrations ${ }^{37}$. Similar results were found in other studies of meropenem $14,16,36,37,39$.

We found that the duration of infusion had a complex effect on the improvement of PTA. It was significant only when using the traditional target of $40 \% \mathrm{fT}>\mathrm{MIC}$ with MIC $<4$ (Fig. 4). For the PD effects of $100 \% \mathrm{fT}>\mathrm{MIC}$ and $100 \% \mathrm{fT}>4 \mathrm{MIC}$, no significant statistical differences were discovered. This finding suggests that, for patients with sensitivity to meropenem and mild infection, prolonging the infusion time can improve the therapeutic effect $(\mathrm{MIC}<4)$. By contrast, those with meropenem-resistance or severe infections (who require a higher therapeutic target) had no significant clinical benefit from prolonged infusion time. A. baumannii and $K$. pneumoniae generally have high MICs. Therefore, higher dosages are needed to achieve the targeted therapeutic effect. However, Mohd et al. conducted an observational study of 211 patients receiving piperacillin/tazobactam and meropenem and found that administration of meropenem by prolonged infusion in critically ill patients was beneficial. Several studies showed similar results and encouraged extended infusions because this maximizes the likelihood of achieving target blood concentrations ${ }^{37,39-42}$.

The reason for this distinction is most likely that few studies have compared the differences in therapeutic responses of $100 \% \mathrm{fT}>\mathrm{MIC}, 100 \% \mathrm{fT}>4 \mathrm{MIC}$, and $40 \% \mathrm{fT}>\mathrm{MIC}$ caused by infusion time; we did so and identified the distinction. It is worth noting that De Waele et al. mentioned that, in a significant subpopulation of critically ill patients with normal renal function, a $100 \% \mathrm{fT}>\mathrm{MIC}$ target is not reached, even with 3-hour extended infusions. This finding agrees with our results.

We also assessed the achievement of different PK/PD target (40\% fT > MIC,100\% fT > MIC and 100\% fT > $4 \mathrm{MIC}$ ) under $\mathrm{MIC}$ values ranging from $1 \mathrm{mg} / \mathrm{L}$ to $8 \mathrm{mg} / \mathrm{L}$. The effect of meropenem dosage and infusion duration was also assessed. In particular, patients with creatinine clearance of $60 \mathrm{ml} / \mathrm{min}$ or less and uric acid greater than $400 \mu \mathrm{mol} / \mathrm{L}$ can achieve the target of PTA $90 \%$ under the MIC of $8 \mathrm{mg} / \mathrm{L}$, even with the administration dose of $500 \mathrm{mg} / 8 \mathrm{~h}$ with a 2-h infusion (Additional file 2). This finding suggests that 500 $\mathrm{mg} / 8 \mathrm{~h}$ is sufficient for critically ill renal failure patients with high uric acid levels.

Although the correlation of antibiotic CL with creatinine clearance has been widely reported ${ }^{11,12,16}$, this study represents the first finding of uric acid having a significant impact on meropenem use. We also found that patients with lower creatinine clearance and high uric acid levels tend to require lower dosages. Our findings suggest that dose adjustment based on these two factors appears to be reasonable.

However, our study also has some limitations. First, the sample size is small, and it is only a single-center study. Second, adverse effects and the influence of plasma concentration were not assessed. Therefore, 
actual tissue concentrations are unknown ${ }^{26,43}$. Measurements of concentrations in the epithelial lining fluid of the lung are needed in further studies ${ }^{37,44}$. In addition, most of the samples were collected at the trough concentration time; this may affect the fitting of the model and intraindividual variability during the treatment period that could not be measured 26,45 .

Nevertheless, our study still provides essential information about the optimized dosage regimen of meropenem in critically ill patients. During empirical therapy of severe pneumonia caused by gramnegative bacteria, clinicians should consider both the achievement of clinical cure and the prevention of drug resistance. Therapeutic drug monitoring is one of the best means to achieve precision therapy.

\section{Conclusions}

Lower CG-CLCR and higher uric acid levels were likely to achieve higher exposure in serum and associate with lower PTA. The dose of $500 \mathrm{mg} / 8 \mathrm{~h}$ may be necessary to achieve an optimal coverage in critically ill patients for all susceptible isolates (MIC $\leq 8 \mathrm{mg} / \mathrm{L}$ ) in patients with high uric acid levels associated with severe renal injury. Moreover, for those with drug-resistant or severe infections (MIC $>4 \mathrm{mg} / \mathrm{L}$ and critically ill patients who need a higher therapeutic target (100\% fT > MIC, $100 \% \mathrm{fT}>4 \mathrm{MIC})$, prolonged infusion time does not appear to be beneficial. Increasing dose or alternative therapeutic strategies may be required for critically ill patients with drug-resistant or severe infections who need a higher therapeutic target.

\section{Abbreviations}

APACHE: Acute Physiology and Chronic Health Evaluation; CG-CLCR: creatinine clearance rate calculated by the equation of Cockcroft-Gault; fT>MIC: Percentage of time remaining concentration above MIC; ICU: Intensive care unit; MDR: Multidrug-resistant; MIC: Minimal inhibitory concentration; attainment; OFV: the objective function values; $\triangle \mathrm{OFV}$ : change of the objective function values; PD: Pharmacodynamic; PK: Pharmacokinetic; PPK: Population pharmacokinetic; PTA: Probability of target.

\section{Declarations}

\section{Acknowledgements:}

The authors would like to acknowledge the support from Hunan Pharmaceutical Association of China and the help of clinicians in the department of Intensive care unit.

\section{Authors' contributions}

YZ and YWX conceived of the study, and participated in its design. YCZ and MY made substantial contributions to data collection, sample and data analysis, and drafting of the manuscript. $\mathrm{HL}$ gave some advice on interpretation of the data. FW contributed in the part of therapeutic drug monitoring. BKZ, DXX 
and FY made contributions in revising the manuscript. All authors read and approved the final manuscript.

\section{Funding}

This study was supported by Hunan Pharmaceutical Association of China with fund number of [HMA202001002].

\section{Availability of data and materials}

The datasets used and analyzed during the current study are available from the corresponding author on reasonable request.

\section{Clinical trials, ethics approval and consent to participate}

Ethics approval was obtained from the local ethics committee (he Second Xiang-ya Hospital of Central South University; approval No. 2019-005). It was also registered in the China Clinical Trial (No.

ChiCTR1900020672). Written informed consent was obtained either from the patient or their appointed legal guardian.

\section{Consent for publication}

Not applicable.

\section{Competing interests}

The authors declare that they have no competing interests.

\section{Author details}

aDepartment of Pharmacy, the Second Xiangya Hospital, Central South University, Changsha, Hunan, P.R. China 410011;

${ }^{b}$ Department of Pharmacy, Xiangtan Central Hospital, Xiangtan, Hunan, P.R. China 411100;

'Department of Basic Medicine and Clinical Pharmacy, China Pharmaceutical University, Nanjing, Jiangsu, P.R. China 210000.

dDepartment of Respiratory and Intensive Care Unit, the Second Xiangya Hospital, Central South University, Changsha, Hunan, P.R. China 410011;

eDepartment of Respiratory and Intensive Care Unit, the Second Xiangya Hospital, Central South University, Changsha, Hunan, P.R. China 410011; 
${ }^{*}$ Corresponding Authors: Department of Pharmacy, the Second Xiangya Hospital, Central South University; Changsha, Hunan, P.R. 410011; China.

Tel: +86-073185292098;

Fax: +86-073184436720.

E-mail addresses: yanmiao@csu.edu.cn.

\section{Statement}

This article is present on a preprint server of Research square website and can be accessed on https://www.researchsquare.com/article/rs-471732/v1. This article is not published nor is under publication elsewhere.

\section{References}

1. Wongsurakiat P, Chitwarakorn N. Severe community-acquired pneumonia in general medical wards: outcomes and impact of initial antibiotic selection. BMC pulmonary medicine 2019; 19: 179.

2. Lanks CW, Musani Al, Hsia DW. Community-acquired Pneumonia and Hospital-acquired Pneumonia. The Medical clinics of North America 2019; 103: 487-501.

3. Chahin A, Opal SM. Severe Pneumonia Caused by Legionella pneumophila: Differential Diagnosis and Therapeutic Considerations. Infectious disease clinics of North America 2017; 31: 111-21.

4. Marti C, Garin N, Grosgurin $\mathrm{O}$ et al. Prediction of severe community-acquired pneumonia: a systematic review and meta-analysis. Critical care (London, England) 2012; 16: R141.

5. Hraiech S, Alingrin J, Dizier S et al. Time to intubation is associated with outcome in patients with community-acquired pneumonia. PloS one 2013; 8: e74937.

6. Restrepo MI, Mortensen EM, Rello $\mathrm{J}$ et al. Late admission to the ICU in patients with communityacquired pneumonia is associated with higher mortality. Chest 2010; 137: 552-7.

7. Restrepo MI, Mortensen EM, Velez JA et al. A comparative study of community-acquired pneumonia patients admitted to the ward and the ICU. Chest 2008; 133: 610-7.

8. Wiseman LR, Wagstaff AJ, Brogden RN et al. Meropenem. A review of its antibacterial activity, pharmacokinetic properties and clinical efficacy. Drugs 1995; 50: 73-101.

9. Dhillon S. Meropenem/Vaborbactam: A Review in Complicated Urinary Tract Infections. Drugs 2018; 78: 1259-70. 
10. Jaruratanasirikul S, Thengyai S, Wongpoowarak W et al. Population pharmacokinetics and Monte Carlo dosing simulations of meropenem during the early phase of severe sepsis and septic shock in critically ill patients in intensive care units. Antimicrobial agents and chemotherapy 2015; 59: 29953001.

11. Ramon-Lopez A, Allen JM, Thomson AH et al. Dosing regimen of meropenem for adults with severe burns: a population pharmacokinetic study with Monte Carlo simulations. The Journal of antimicrobial chemotherapy 2015; 70: 882-90.

12. Zhou QT, He B, Shen N et al. Meropenem Dosing Based on a Population PharmacokineticPharmacodynamic Model in Elderly Patients with Infection of the Lower Respiratory Tract. Drugs \& aging 2017; 34: 115-21.

13. Conte JE, Jr., Golden JA, Kelley MG et al. Intrapulmonary pharmacokinetics and pharmacodynamics of meropenem. International journal of antimicrobial agents 2005; 26: 449-56.

14. Cies JJ, Moore WS, 2nd, Enache A et al. Population Pharmacokinetics and Pharmacodynamic Target Attainment of Meropenem in Critically III Young Children. The journal of pediatric pharmacology and therapeutics : JPPT : the official journal of PPAG 2017; 22: 276-85.

15. Usman M, Frey OR, Hempel G. Population pharmacokinetics of meropenem in elderly patients: dosing simulations based on renal function. European journal of clinical pharmacology 2017; 73: 333-42.

16. Alobaid AS, Wallis SC, Jarrett P et al. Effect of Obesity on the Population Pharmacokinetics of Meropenem in Critically III Patients. Antimicrobial agents and chemotherapy 2016; 60: 4577-84.

17. Doh K, Woo H, Hur J et al. Population pharmacokinetics of meropenem in burn patients. The Journal of antimicrobial chemotherapy 2010; 65: 2428-35.

18. Hanberg P, Öbrink-Hansen K, Thorsted A et al. Population Pharmacokinetics of Meropenem in Plasma and Subcutis from Patients on Extracorporeal Membrane Oxygenation Treatment. Antimicrobial agents and chemotherapy 2018; 62.

19. Kristoffersson AN, David-Pierson P, Parrott NJ et al. Simulation-Based Evaluation of PK/PD Indices for Meropenem Across Patient Groups and Experimental Designs. Pharmaceutical research 2016; 33: $1115-25$.

20. Roberts JA, Abdul-Aziz MH, Lipman J et al. Individualised antibiotic dosing for patients who are critically ill: challenges and potential solutions. The Lancet Infectious diseases 2014; 14: 498-509.

21. Mendez A, Chagastelles P, Palma E et al. Thermal and alkaline stability of meropenem: degradation products and cytotoxicity. International journal of pharmaceutics 2008; 350: 95-102. 
22. Jaruratanasirikul S, Sriwiriyajan S. Comparison of the pharmacodynamics of meropenem in healthy volunteers following administration by intermittent infusion or bolus injection. The Journal of antimicrobial chemotherapy 2003; 52: 518-21.

23. McKinnon PS, Paladino JA, Schentag JJ. Evaluation of area under the inhibitory curve (AUIC) and time above the minimum inhibitory concentration ( $T>M I C)$ as predictors of outcome for cefepime and ceftazidime in serious bacterial infections. International journal of antimicrobial agents 2008; 31 : 345-51.

24. Tam VH, McKinnon PS, Akins RL et al. Pharmacodynamics of cefepime in patients with Gramnegative infections. The Journal of antimicrobial chemotherapy 2002; 50: 425-8.

25. Abdul-Aziz MH, Lipman J, Akova M et al. Is prolonged infusion of piperacillin/tazobactam and meropenem in critically ill patients associated with improved pharmacokinetic/pharmacodynamic and patient outcomes? An observation from the Defining Antibiotic Levels in Intensive care unit patients (DALI) cohort. The Journal of antimicrobial chemotherapy 2016; 71: 196-207.

26. Veiga RP, Paiva JA. Pharmacokinetics-pharmacodynamics issues relevant for the clinical use of beta-lactam antibiotics in critically ill patients. Critical care (London, England) 2018; 22: 233.

27. Shahbaz H, Gupta M. Creatinine Clearance. StatPearls. Treasure Island (FL): StatPearls Publishing

Copyright @ 2021, StatPearls Publishing LLC., 2021.

28. Nunes MBG, Filho AC, Alvares VRC et al. CKD-EPI versus Cockcroft-Gault formula for predicting contrast-induced nephropathy following percutaneous coronary intervention in patients without significant renal impairment. Revista portuguesa de cardiologia : orgao oficial da Sociedade Portuguesa de Cardiologia = Portuguese journal of cardiology : an official journal of the Portuguese Society of Cardiology 2018; 37: 25-33.

29. Casals G, Hernández C, Hidalgo S et al. Development and validation of a UHPLC diode array detector method for meropenem quantification in human plasma. Clinical biochemistry 2014; 47: 223-7.

30. Zou L, Meng F, Hu L et al. A novel reversed-phase high-performance liquid chromatographic assay for the simultaneous determination of imipenem and meropenem in human plasma and its application in TDM. Journal of pharmaceutical and biomedical analysis 2019; 169: 142-50.

31. Wong G, Farkas A, Sussman R et al. Comparison of the accuracy and precision of pharmacokinetic equations to predict free meropenem concentrations in critically ill patients. Antimicrobial agents and chemotherapy 2015; 59: 1411-7.

32. Zhao W, Kaguelidou F, Biran V et al. External Evaluation of Population Pharmacokinetic Models of Vancomycin in Neonates: The transferability of published models to different clinical settings. British 
journal of clinical pharmacology 2013; 75: 1068-80.

33. Glen JB, Servin F. Evaluation of the predictive performance of four pharmacokinetic models for propofol. British journal of anaesthesia 2009; 102: 626-32.

34. Egi $\mathrm{A}$, Fukuda $\mathrm{H}$, Kawamoto $\mathrm{M}$ et al. [Preoperative prediction of creatinine clearance by using serum creatinine]. Masui The Japanese journal of anesthesiology 2004; 53: 1306-10.

35. Chung EK, Cheatham SC, Fleming MR et al. Population Pharmacokinetics and Pharmacodynamics of Meropenem in Nonobese, Obese, and Morbidly Obese Patients. Journal of clinical pharmacology 2017; 57: 356-68.

36. Sjövall F, Alobaid AS, Wallis SC et al. Maximally effective dosing regimens of meropenem in patients with septic shock. The Journal of antimicrobial chemotherapy 2018; 73: 191-8.

37. Benítez-Cano A, Luque S, Sorlí L et al. Intrapulmonary concentrations of meropenem administered by continuous infusion in critically ill patients with nosocomial pneumonia: a randomized pharmacokinetic trial. Critical care (London, England) 2020; 24: 55.

38. Burger R, Guidi M, Calpini V et al. Effect of renal clearance and continuous renal replacement therapy on appropriateness of recommended meropenem dosing regimens in critically ill patients with susceptible life-threatening infections. The Journal of antimicrobial chemotherapy 2018; 73: 3413-22.

39. Corcione S, D'Avolio A, Loia RC et al. Pharmacokinetics of meropenem in burn patients with infections caused by Gram-negative bacteria: Are we getting close to the right treatment? Journal of global antimicrobial resistance 2020; 20: 22-7.

40. Wunderink RG, Matsunaga Y, Ariyasu M et al. Cefiderocol versus high-dose, extended-infusion meropenem for the treatment of Gram-negative nosocomial pneumonia (APEKS-NP): a randomised, double-blind, phase 3, non-inferiority trial. The Lancet Infectious diseases 2021; 21: 213-25.

41. Nguyen CP, Dan Do TN, Bruggemann R et al. Clinical cure rate and cost-effectiveness of carbapenem-sparing beta-lactams vs. meropenem for Gram-negative infections: A systematic review, meta-analysis, and cost-effectiveness analysis. International journal of antimicrobial agents 2019; 54: 790-7.

42. Ehmann L, Zoller M, Minichmayr IK et al. Development of a dosing algorithm for meropenem in critically ill patients based on a population pharmacokinetic/pharmacodynamic analysis. International journal of antimicrobial agents 2019; 54: 309-17.

43. Roberts JA, Udy AA, Jarrett $P$ et al. Plasma and target-site subcutaneous tissue population pharmacokinetics and dosing simulations of cefazolin in post-trauma critically ill patients. The Journal of antimicrobial chemotherapy 2015; 70: 1495-502. 
44. Motos A, Kuti JL, Li Bassi G et al. Is One Sample Enough? $\beta$-Lactam Target Attainment and Penetration into Epithelial Lining Fluid Based on Multiple Bronchoalveolar Lavage Sampling Time Points in a Swine Pneumonia Model. Antimicrobial agents and chemotherapy 2019; 63.

45. Neely MN, van Guilder MG, Yamada WM et al. Accurate detection of outliers and subpopulations with Pmetrics, a nonparametric and parametric pharmacometric modeling and simulation package for R. Therapeutic drug monitoring 2012; 34: 467-76.

\section{Figures}

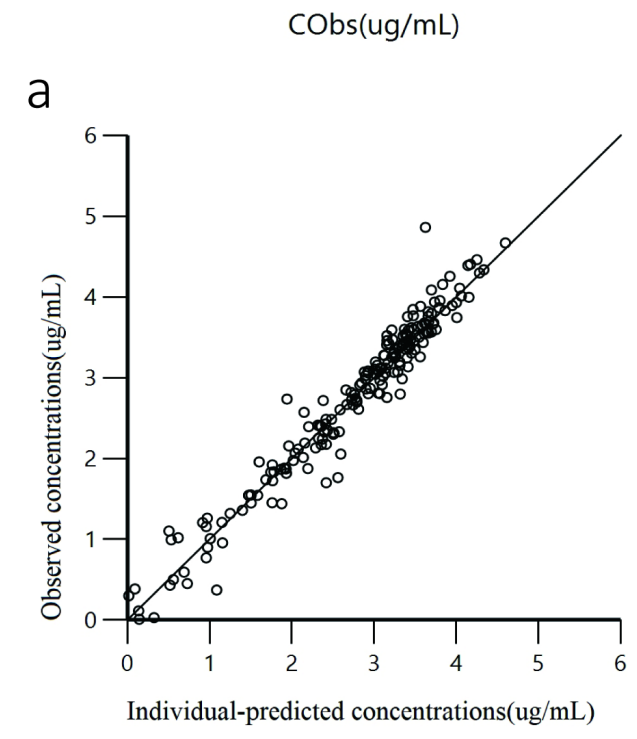

$\operatorname{CObs}(\mathrm{ug} / \mathrm{mL})$

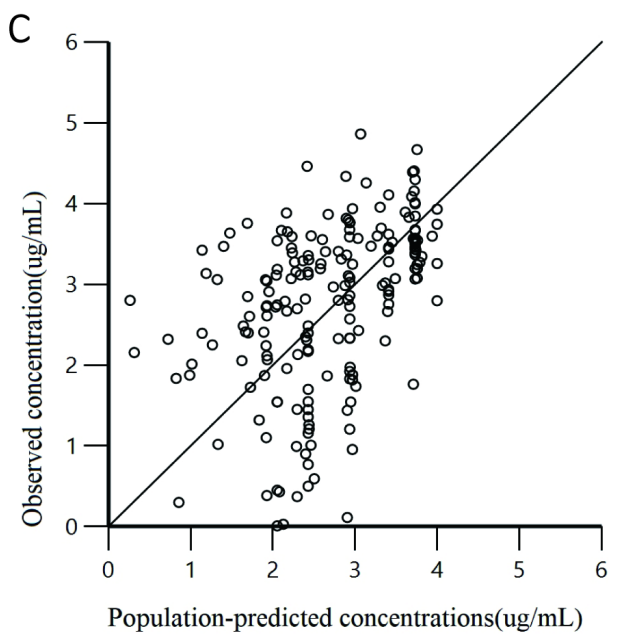

cstep17 V2-UAumolL CI-UAumolL CI-CLcrCGmImin, $\operatorname{CObs}(\mathrm{ug} / \mathrm{mL})$

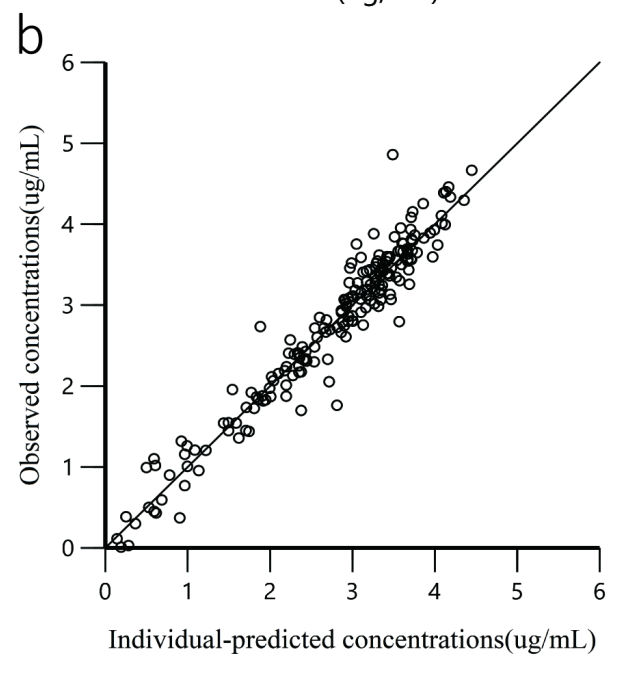

cstep17 V2-UAumolL Cl-UAumolL Cl-CLcrCGmimin, $\operatorname{CObs}(\mathrm{ug} / \mathrm{mL})$

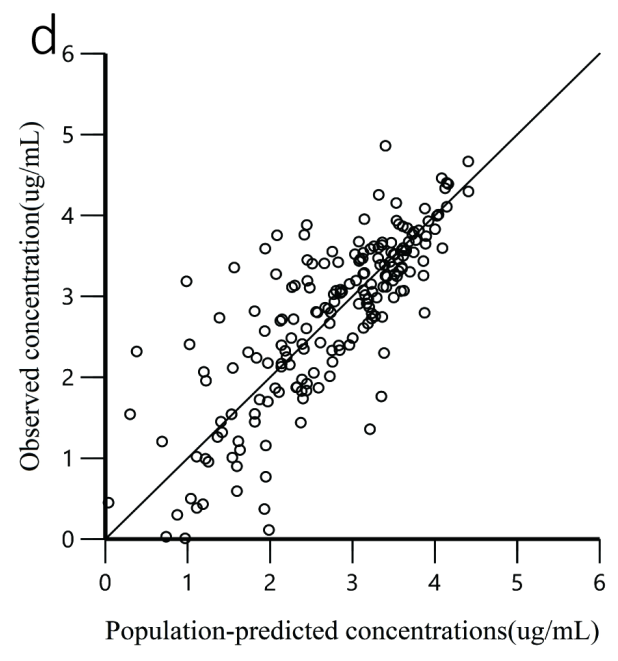

\section{Figure 1}


Scatter plots of DV-PRED and DV-IPRED in basic model and final model Individual ( $a$ and $b$ ) and population ( $c$ and $d$ ) predicted meropenem concentrations vs observed concentrations of meropenem for the basic model ( $a$ and $c$ ) and the final model ( $c$ and $d$ ).

a

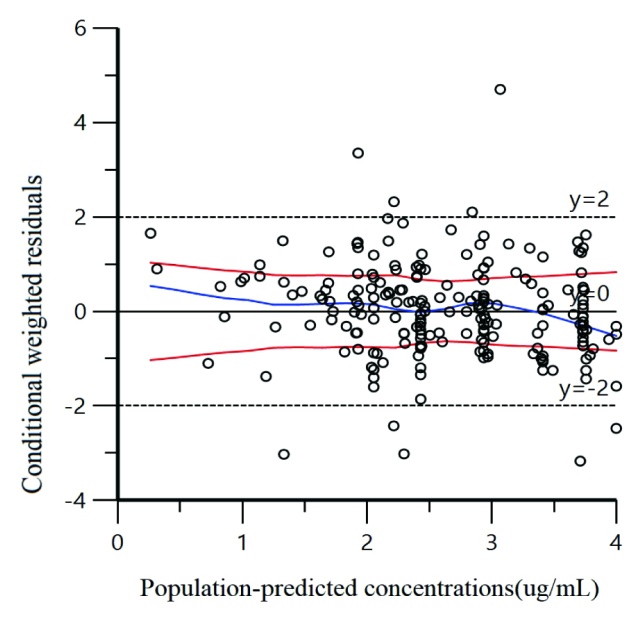

C

$\operatorname{CObs}(\mathrm{ug} / \mathrm{mL})$

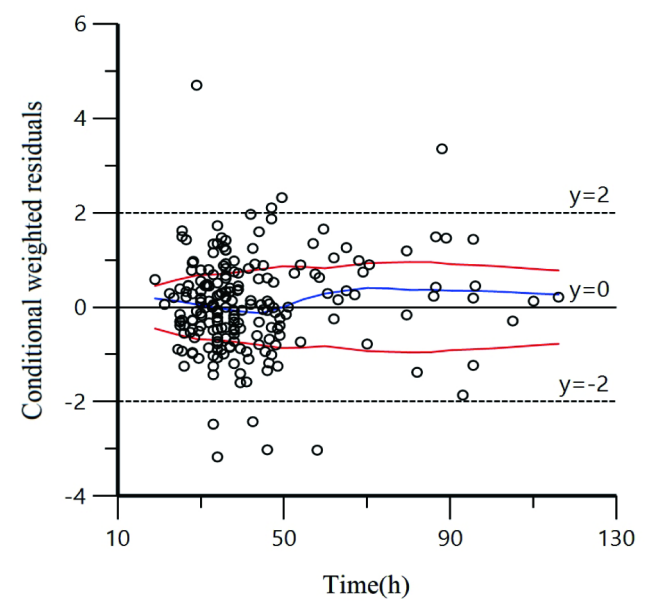

b cstep17 V2-UAumolL Cl-UAumolL CI-CLcrCGmImin, $\operatorname{CObs}(\mathrm{ug} / \mathrm{mL})$

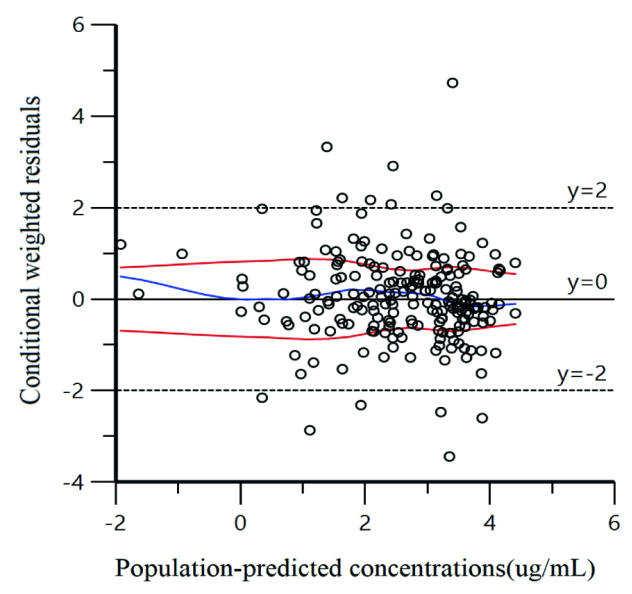

cstep17 V2-UAumolL Cl-UAumolL Cl-CLcrCGmImin, $\operatorname{CObs}(\mathrm{ug} / \mathrm{mL})$

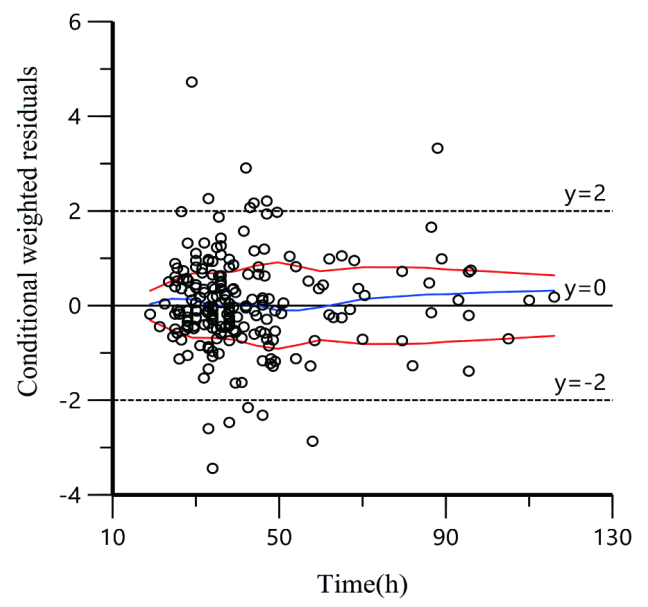

Figure 2

Scatter plots of conditional weighted residuals in basic model and final model The distribution of conditional weighted residuals for the basic model ( $a$ and $c$ ) and the final model ( $b$ and $d)$. The conditional weighted residuals versus population-predicted concentrations ( $a$ and $b$ ) and time ( $c$ and $d$ ). 
a

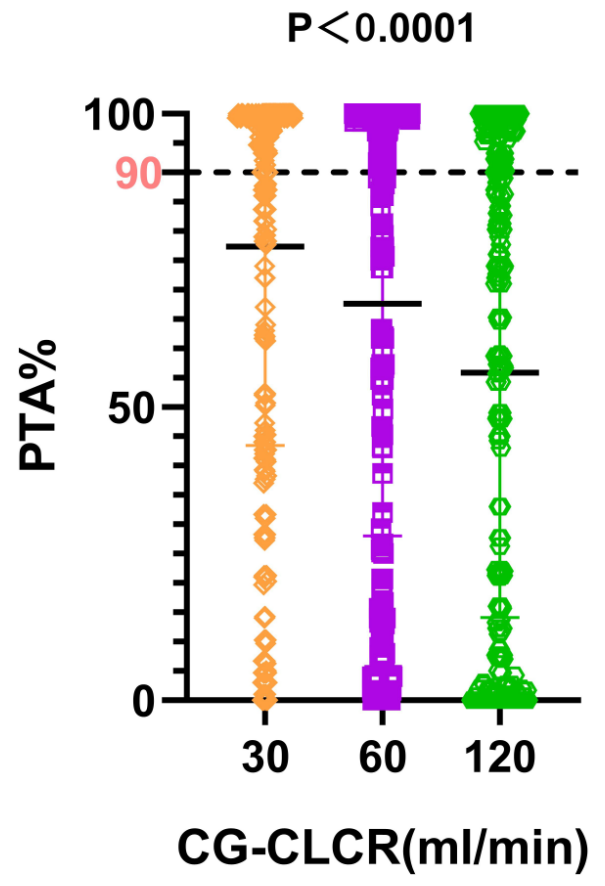

b

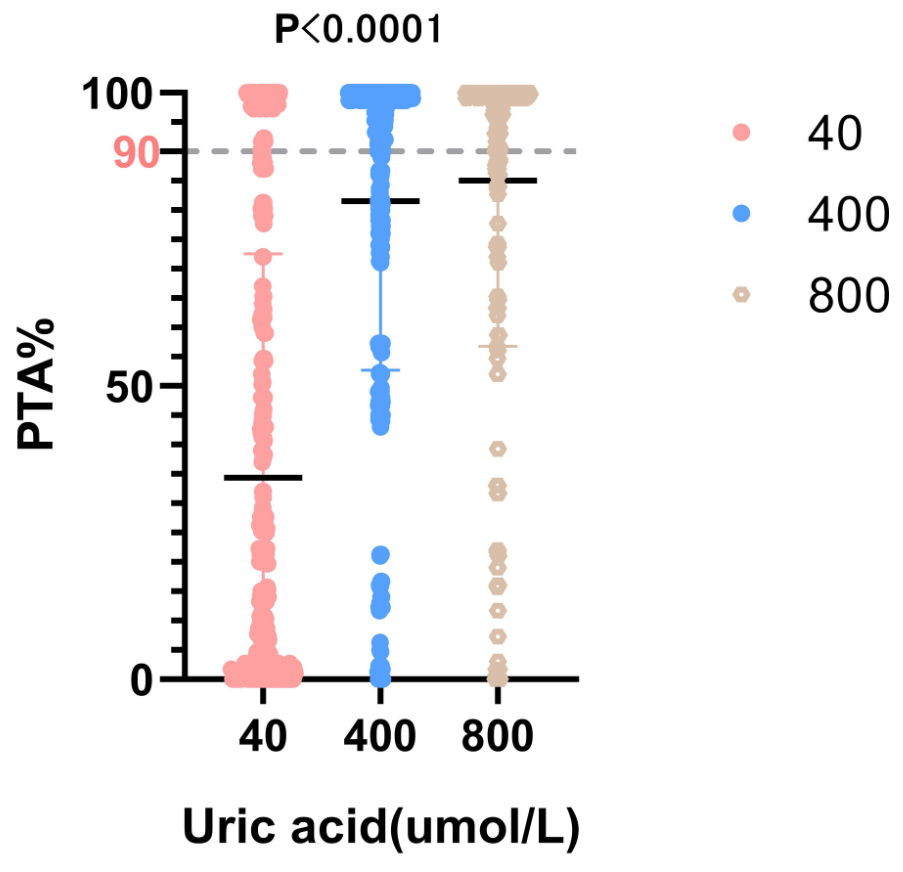

Figure 3

The effect of CGCL-CR and uric acid to PTA. Distinctions of PTA in different CG-CLCR (a) and uric acid (b) group based on the simulated data.

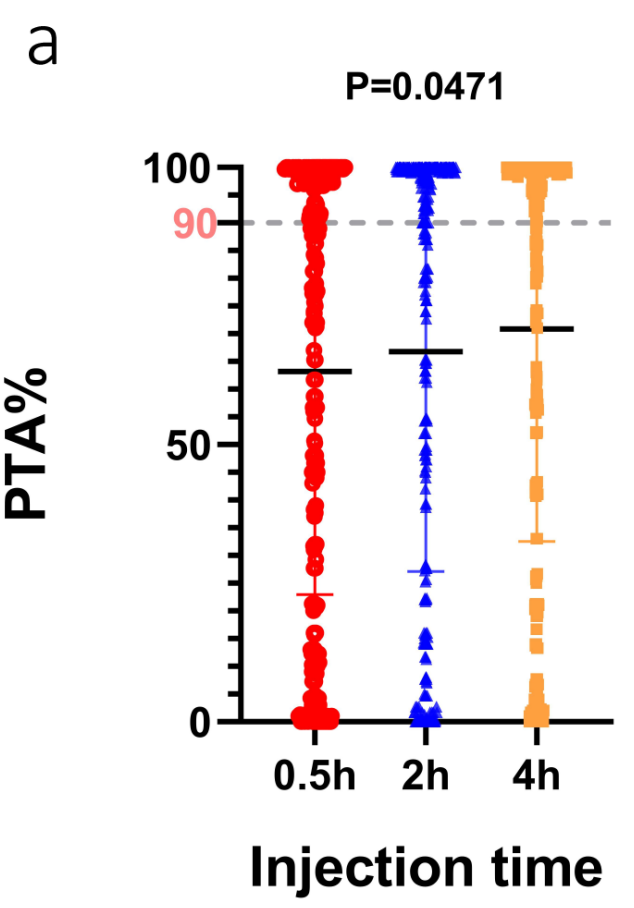

b

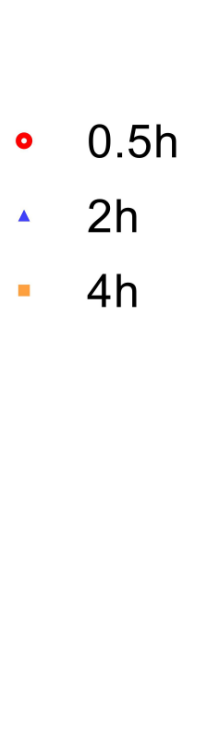

$4 \mathrm{~h}$

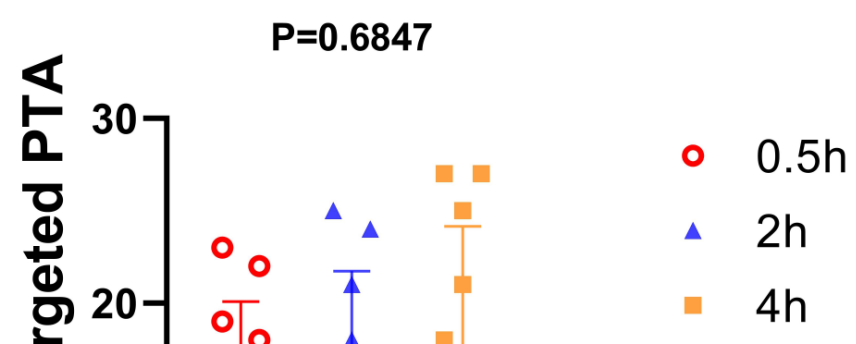

Injection time

Figure 4 
The effect of injection time to PTA Distinctions of PTA (a) and the number of achieved targeted PTA $\geq 90 \%(b)$ in different injection time $(0.5 \mathrm{~h}, 2 \mathrm{~h}$ and $4 \mathrm{~h})$ group.
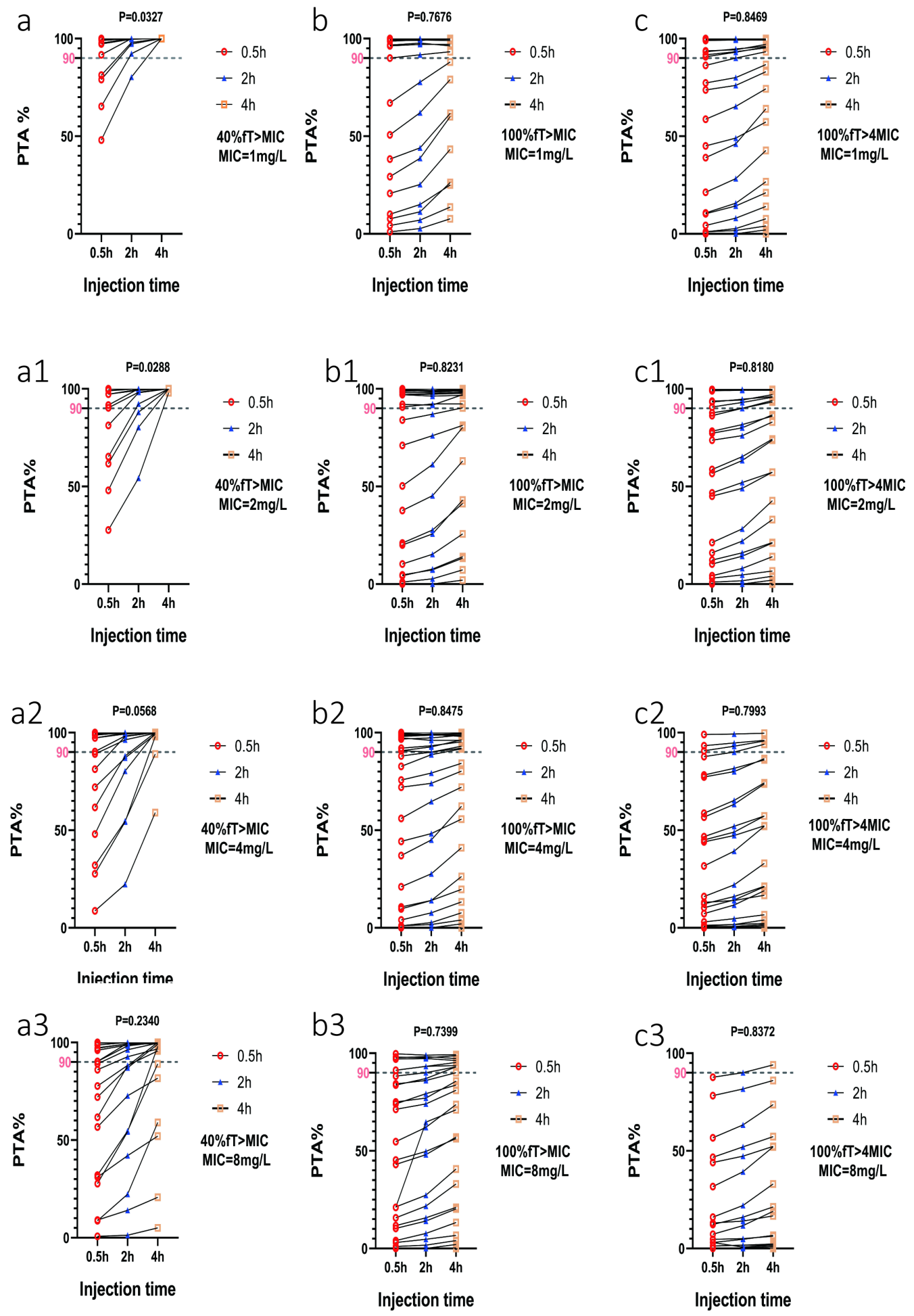

Figure 5

The effect of infusion time to PTA under different MICs. Distinctions of injection time $(0.5 \mathrm{~h}, 2 \mathrm{~h}$ and $4 \mathrm{~h})$ on simulated PTA value in $\mathrm{MIC}=1 \mathrm{mg} / \mathrm{L}(\mathrm{a}, \mathrm{b}, \mathrm{c}), \mathrm{MIC}=2 \mathrm{mg} / \mathrm{L}(\mathrm{a} 1, \mathrm{~b} 1$ and $\mathrm{c} 1), \mathrm{MIC}=4 \mathrm{mg} / \mathrm{L}(\mathrm{a} 2, \mathrm{~b} 2$ and $\mathrm{c} 2)$ and $\mathrm{MIC}=8 \mathrm{mg} / \mathrm{L}(\mathrm{a} 3, \mathrm{~b} 3$ and c3) groups, respectively. Distinctions of injection time $(0.5 \mathrm{~h}, 2 \mathrm{~h}$ and $4 \mathrm{~h})$ on 
simulated PTA value for PK/PD target of $40 \% \mathrm{fT}>\mathrm{MIC}(\mathrm{a}, \mathrm{a} 1, \mathrm{a} 2$ and $\mathrm{a} 3), 100 \% \mathrm{fT}>\mathrm{MIC}(\mathrm{b}, \mathrm{b} 1, \mathrm{~b} 2$ and $\mathrm{b} 3)$ and $100 \%$ fT $>4 \mathrm{MIC}(\mathrm{c}, \mathrm{c} 1, \mathrm{c} 2$ and $\mathrm{c} 3)$, respectively.

\section{Supplementary Files}

This is a list of supplementary files associated with this preprint. Click to download.

- Additionalfile1.pdf

- Additionalfile2.pdf

- Additionalfile3.pdf 